\title{
Neostigmine: A Safe and Simple Solution to Acute Colonic Pseudo-Obstruction.
}

\author{
Gutiérrez I, Vicol A, Roldán N, Vendrell M, Deiros C, Masdeu J. Consorci Sanitari Integral, Barcelona.
}

INTRODUCTION: Acute colonic pseudo-obstruction (ACPO), or Ogilvie syndrome, is characterized by a massive colonic distension without mechanical obstruction. Pathophysiology is unclear, being mainly an altered intestinal motility due to a disorder of the autonomous sytem. Prompt treatment and resolution are prioritary. In this case report medical treatment was performed, based on physiology and pharmacology.

CASE REPORT: A 40-year-old man, with a history of spastic tetraparesis due to cerebral palsy and chronic constipation requiring weekly enemas, was admitted to the hospital after 3 days of general discomfort without fever. Dehydration, bloating and a distended abdomen were noted, with no hemodynamic or respiratory instability. Blood tests were normal. Abdominal x-ray revealed significant colonic dilatation. Due to scarce improvement with conservative treatment, and a control $x$-ray showing further small bowel and gastric distention, neostigmine was indicated. Under standard monitorisation and with ready access to advanced resuscitation equipment, neostigmine was titrated according to clinical response up to a total of $3.5 \mathrm{mg}$. Progressive bradycardia from 78 to $47 \mathrm{bpm}$ with no hemodynamic instability was observed, with an increase of salivary secretions. Effectivity was noted by the passing of two pasty stools. After 2 days, nasogastric tube could be removed, with correct oral intake and radiological improvement.

DISCUSSION: ACPO is thought to be due to a deficiency in the parasympathetic autonomic nervous

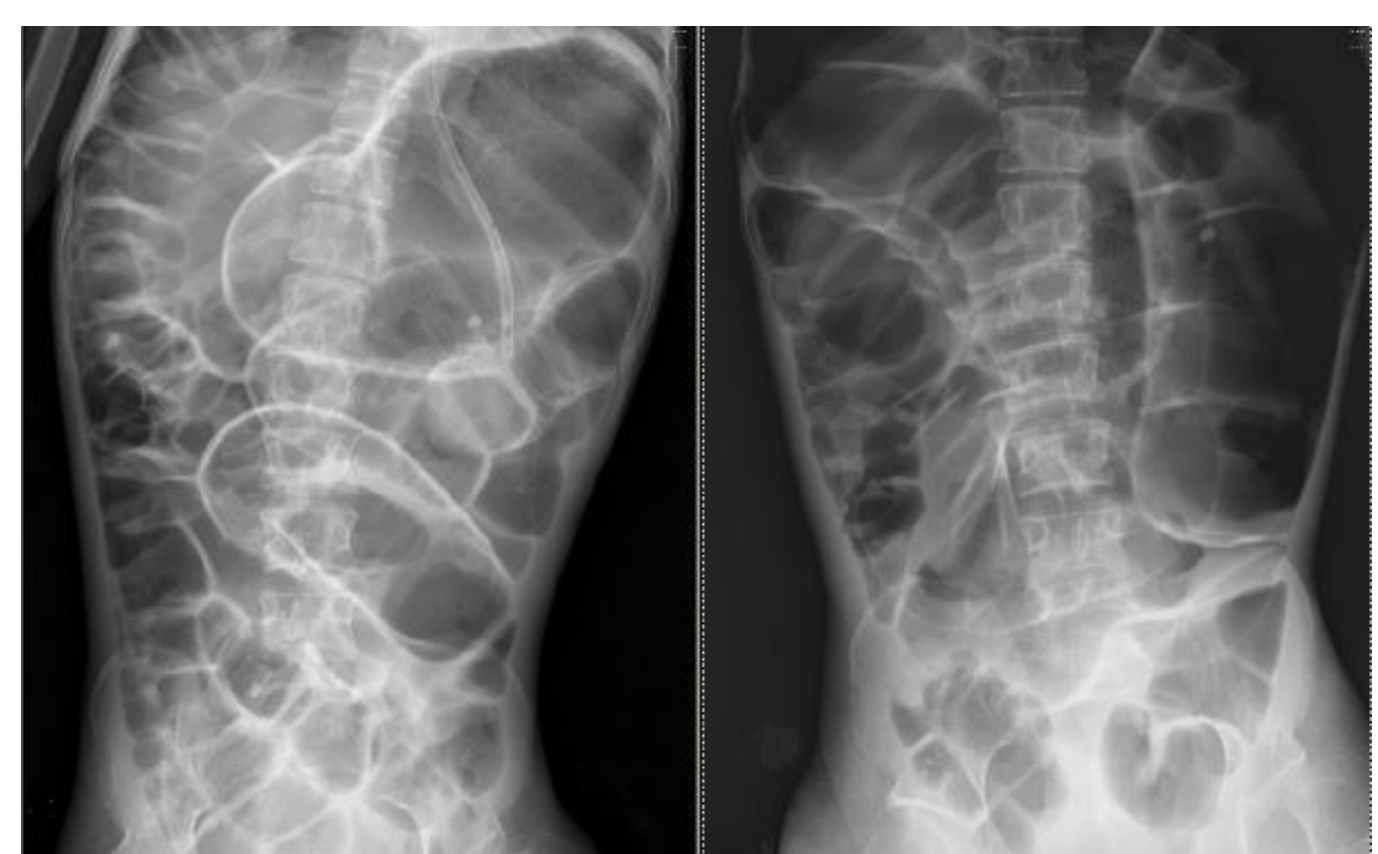
system, responsible of gut inervation. Neurological diseases, as in our case, have been associated to this syndrome. The most feared complication is cecal perforation and peritonitis, associated with mortality rate close to $70 \%$.

Initial treatment is conservative, including bowel rest, opioid discontinuation, correction of electrolyte imbalances and underlying infection, and nasogastric and rectal tubes. Neostigmine increases the amount of available acetylcholine, indirectly stimulating muscarinic receptors in the smooth muscle of the digestive system, causing contraction. However, side effects are not restricted to the intestine, being the most important the risk of bradycardia and cardiac arrest, thus strict monitoring is required.

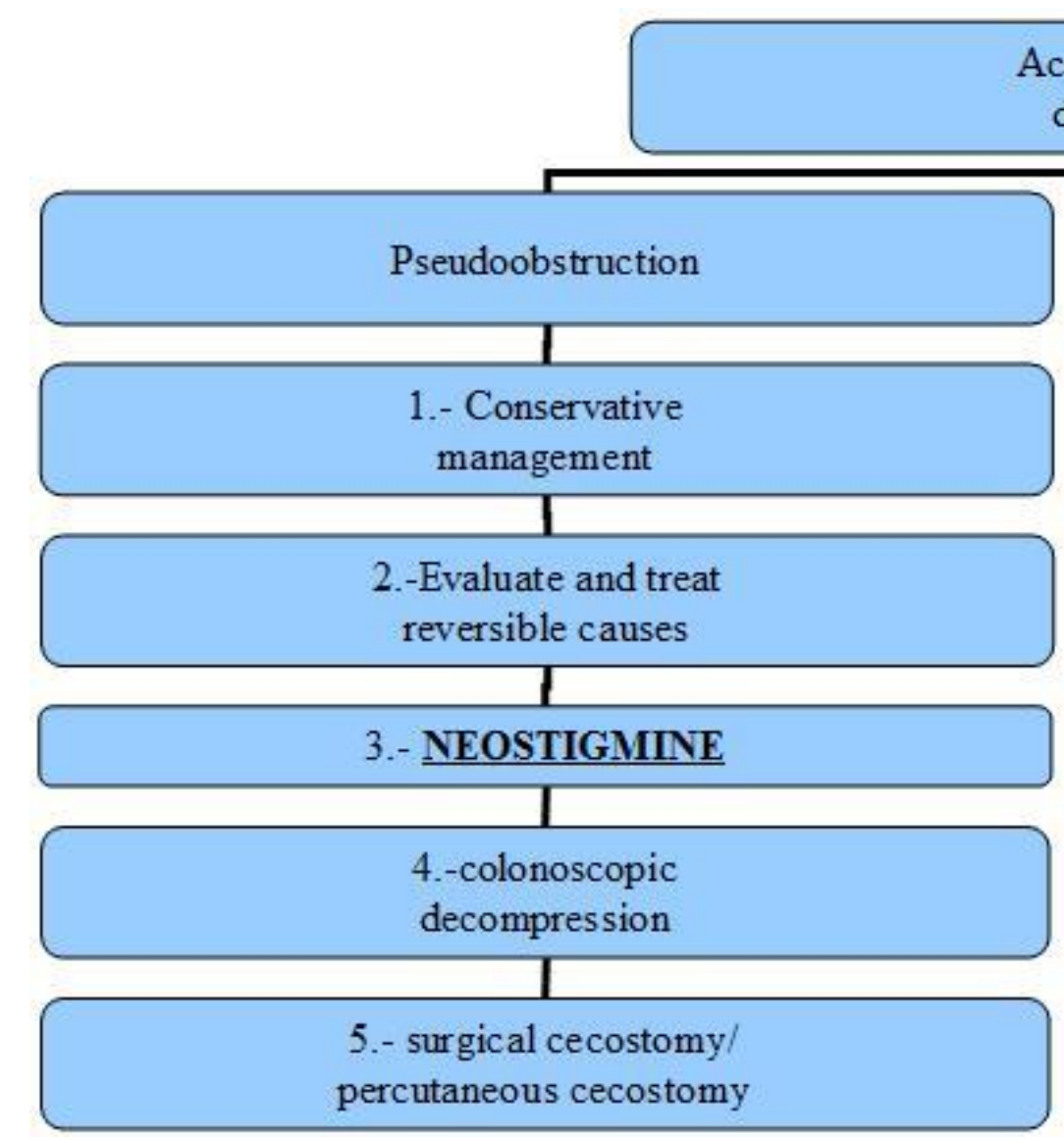

CONCLUSION: Monitored use of neostigmine, is a simple, safe, and effective treatment for ACPO. 